Research Article

\title{
The Effects of Alternate-Day Corticosteroids in Autoimmune Disease Patients
}

\author{
Genny Margarita Chaia-Semerena, María Eugenia Vargas-Camaño, \\ Cesar Daniel Alonso-Bello (iD, Jorge Javier Guillén-Toledo, \\ Ricardo Leopoldo Guido-Bayardo, Fernando Lozano-Patiño, \\ Mariano Daniel Temix-Delfín, and María Isabel Castrejón-Vázquez
}

\begin{abstract}
Clinical Immunology and Allergy Service, National Medical Center 20 de Noviembre, Instituto de Seguridad y Servicios Sociales de Los Trabajadores del Estado, México City, Mexico

Correspondence should be addressed to Cesar Daniel Alonso-Bello; cesar_alonso86@hotmail.com
\end{abstract}

Received 8 January 2020; Revised 3 April 2020; Accepted 24 April 2020; Published 18 May 2020

Academic Editor: Rizgar Mageed

Copyright (C) 2020 Genny Margarita Chaia-Semerena et al. This is an open access article distributed under the Creative Commons Attribution License, which permits unrestricted use, distribution, and reproduction in any medium, provided the original work is properly cited.

\begin{abstract}
Introduction. Several studiesdemonstrated that the use of alternate-day corticosteroid therapy maintains control of autoimmune diseases due to the prolongation of their therapeutic effect beyond their metabolic effect, with a significant decrease in side effects in patients. For this reason, the current recommendation for the use of these medications is in a short cycle to avoid adverse effects when used frequently and for prolonged periods of time. Objectives. To learn variations in serum levels of autoantibodies in autoimmune diseases treated with steroids on alternate days, as well as whether there are differences in the response to them depending on the type of disease. Study Design. A descriptive, retrospective, and cross-sectional study was conducted in which serum autoantibody levels were compared at the time of diagnosis and three months after alternate-day corticosteroid therapy. Results. We included 106 patients from three autoimmune connective tissue diseases (systemic lupus erythematosus, Sjögren syndrome, and Hashimoto's thyroiditis) and observed a statistically significant decrease in serum autoantibody levels both in patients with lupus and those with Hashimoto's thyroiditis, regardless of the sex of the patients, as well as the type of steroids used. Conclusions. Treatment with alternate-day corticosteroids achieved a statistically significant decrease in serum autoantibody levels in patients with systemic lupus erythematosus and Hashimoto's thyroiditis.
\end{abstract}

\section{Introduction}

Autoimmune diseases are characterized by the loss of control in a $\mathrm{T}$ lymphocyte subgroup with alteration in the differentiation of the own and the foreign [1-4]. When a secondary activation of lymphocytes is associated with the production of antibodies against various antigens of the body, it is called humoral autoimmune disease and, in this case, the treatment involves providing drugs that stop the uncontrolled production of autoantibodies to prevent the perpetuation of organ damage. The medications used for this purpose could trigger side effects that deteriorate the quality of life of patients and are usually dose dependent. Within these, the most used are systemic steroids [5-11]. Several publications have been demonstrated that the use of alternate-day corticosteroid therapy maintains control of autoimmune diseases due to the prolongation of their therapeutic effect beyond their metabolic effect, with a significant decrease in side effects [12-14]. It has been proven that steroid schemes on alternate days have a lower suppressive effect of the hypothalamic-pituitary-adrenal axis compared to treatment with daily intake when evaluated by means of insulin-induced hypoglycemia [15]. There is also known that the steroid scheme on alternate days administered to children with nephrotic syndrome does not affect the expected growth for age [16]. The concentration of the medication on alternate days maintains the serum levels and is associated with reduced suppression of the hypothalamic- 
pituitary-adrenal axis [17]. The current recommendation for the use of systemic steroids is in short cycles and accompanied by other steroid-saving drugs to reduce the side effects of prolonged cycles. In this article, we presented the response observed in serum levels of autoantibodies in patients with autoimmune diseases treated with alternateday corticosteroid therapy between January 2008 and January 2013 at the Clinical Immunology and Allergy Service of the National Medical Center 20 de Noviembre, ISSSTE, Mexico.

\section{Materials and Methods}

A descriptive, retrospective, and cross-sectional study was conducted in which serum autoantibody levels were compared at the time of diagnosis and three months after alternate-day corticosteroid therapy. In the period between January 1, 2008, and January 1, 2013, 318 patients were evaluated for three autoimmune diseases encoded in the ICD10 , and the diseases were systemic lupus erythematosus (SLE), Sjögren's syndrome (SS), and Hashimoto's thyroiditis (HT). 106 patients were selected by the inclusion criteria, selecting those who had at least two autoantibody determinations, one of them prior to steroid treatment on alternate days after the diagnosis of autoimmune disease and the other three months after onset and without another immunosuppressive treatment that could interfere with the immune response. The dose of the steroid used was $0.5-1 \mathrm{mg} / \mathrm{kg}$, calculated based on prednisone or its equivalent dose with deflazacort, and the decision to start treatment was the clinical activity of the autoimmune disease. Groups according to the autoimmune disease were classified. The autoantibodies selected for the diseases studied were anti-ds DNA (double-stranded DNA autoantibodies) for SLE, anti-SSA and anti-SSB for SS, and anti-Tg and anti-TPO (thyroglobulin and thyroid peroxidase) for HT. Subsequently, within the groups formed, they were subclassified according to gender, age, steroid indicated (prednisone or deflazacort), and dose. All autoantibody quantifications were performed in the immunology laboratory by the radioimmunoassay. The statistical analysis was performed in STATISTICA 8.0 and STATA 11 with a descriptive and inferential statistical analysis, and comparisons were made regarding the variables studied (disease, sex, and age) and steroids used. To investigate the strength of association between the nominal variables with a $95 \%$ confidence interval, we used an ANOVA analysis of one, two, and three ways, to compare the means of serum antibody levels before and after initiation of systemic steroid treatment. This retrospective study was approved by the CMN20NOV Institutional Review Board and Ethics Committee.

\section{Results}

A total of 106 patients were analyzed, 17 with SLE, 10 with SS, and 79 with HT with a mean age of $42.5 \pm 17.04$ years. The main features are summarized in Table 1.

Regarding the treatment used due to illness, the distribution was as follows: in SLE, $58.82 \%(n=10)$ with prednisone and $41.18 \%(n=7)$ with deflazacort. In SS, 50\% $(n=5)$
TABLE 1: General characteristics of the patients and classification by diagnosis.

\begin{tabular}{lccccc}
\hline Disease & $N$ & Women & Men & $\begin{array}{c}\text { Age } \\
\text { range }\end{array}$ & Middle age \\
\hline $\begin{array}{l}\text { Demographic characteristics } \\
\text { SLE }\end{array}$ & 17 & 16 & 1 & $11-69$ & $42.5 \pm 17.04$ \\
$\begin{array}{l}\text { Sjögren syndrome } \\
\text { Autoimmune thyroid } \\
\text { disease }\end{array}$ & 79 & 9 & 1 & $60-94$ & $69.8 \pm 10.11$ \\
\hline
\end{tabular}

with prednisone and $50 \%(n=5)$ with deflazacort. Finally, in the HT group, $46.84 \%(n=37)$ with prednisone and $53.16 \%$ $(n=42)$ with deflazacort.

The analysis of results in patients with SLE shows an average of anti-ds DNA antibodies prior to treatment with prednisone of $442.0 \mathrm{IU} / \mathrm{mL}$ and deflazacort $830.6 \mathrm{IU} / \mathrm{mL}$ and posttreatment of $129.8 \mathrm{IU} / \mathrm{mL}$ with prednisone and $179.6 \mathrm{IU} / \mathrm{mL}$ with deflazacort. The analysis of variance shows $p=0.1851$ for the difference in antibody levels between treatments and $p=0.005$ for the difference in pre- and posttreatment values independent of the indicated treatment (Figure 1).

For SS, two antibodies, anti-SSA and anti-SSB, were analyzed. The mean pretreatment values were as follows: for anti-SSA, $71.0 \mathrm{IU} / \mathrm{mL}$ with prednisone and $97.6 \mathrm{IU} / \mathrm{mL}$ with deflazacort, and for anti-SSB, $15.3 \mathrm{IU} / \mathrm{mL}$ with prednisone and $45.0 \mathrm{IU} / \mathrm{mL}$ with deflazacort. After treatment, the values for anti-SSA were $41.6 \mathrm{IU} / \mathrm{mL}$ with prednisone and $36.8 \mathrm{IU} /$ $\mathrm{mL}$ with deflazacort. For anti-SSB, the values were $10.0 \mathrm{IU} /$ $\mathrm{mL}$ for prednisone and $20.4 \mathrm{IU} / \mathrm{ml}$ for deflazacort. The analysis of variance shows $p=0.2546$ for the difference in antibody levels between treatments and $p=0.0311$ for the difference of pre- and posttreatment values independent of the indicated treatment. There is a greater difference between the decrease in anti-SSA antibodies compared to anti-SSB levels with $p=0.0061$ (Figure 2).

For HT, two antibodies, anti-Tg and anti-TPO, were analyzed. The mean pretreatment value of anti-Tg was $240.0 \mathrm{IU} / \mathrm{mL}$ for prednisone and $358.5 \mathrm{UI} / \mathrm{mL}$ for deflazacort and of anti-TPO was $747.3 \mathrm{IU} / \mathrm{mL}$ for prednisone and $601.5 \mathrm{IU} / \mathrm{mL}$ for deflazacort. Posttreatment values are as follows: for anti-Tg, the value was $94.4 \mathrm{IU} / \mathrm{mL}$ for prednisone and $38.7 \mathrm{IU} / \mathrm{mL}$ for deflazacort and for anti-TPO, the value was $183.2 \mathrm{IU} / \mathrm{mL}$ for prednisone and $198.8 \mathrm{IU} / \mathrm{mL}$ for deflazacort. The analysis of variance shows $p=0.7783$ for the difference in antibody levels between treatments and $p \leq 0.0001$ for the level of pre- and posttreatment antibodies. There is an important difference in the decrease between the types of antibodies, and the decrease among the anti-TPOs was greater compared to the anti-Tg with $p=0.000038$ (Figure 3).

\section{Discussion}

In the group of patients studied, three characteristic autoimmune pathologies (SLE, SS, and HT) were included; the number of patients was higher in the HT group due to the 


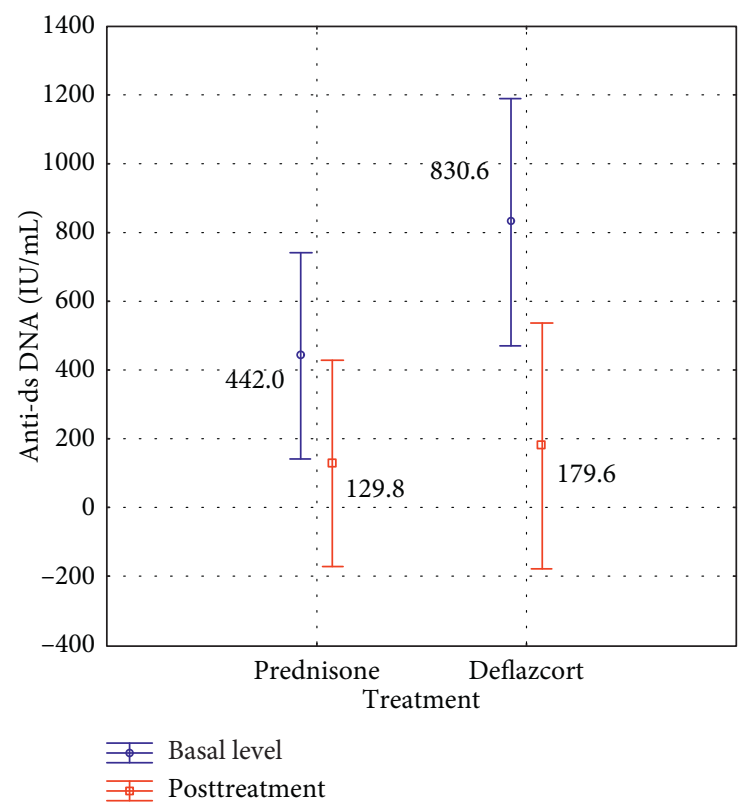

(a)

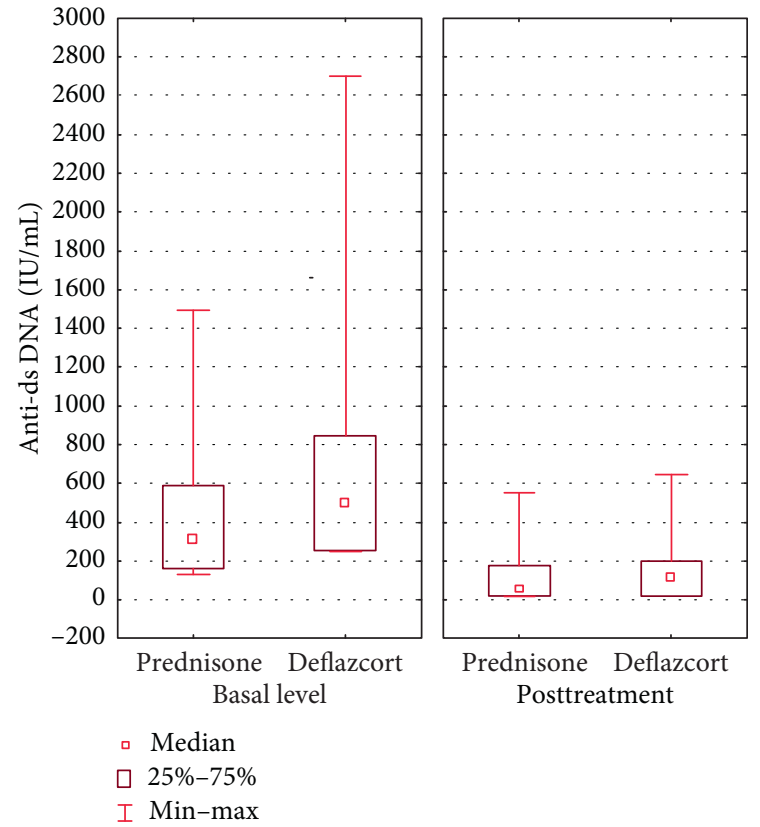

(b)

Figure 1: (a) Variance analysis of the mean in anti-ds DNA antibodies before and after steroid treatment. (b) Comparative analysis of the median in response of serum autoantibody levels, ranges, and interquartile ranges according to the indicated treatment and titration phase. Follow-up was done three months after the initial treatment.

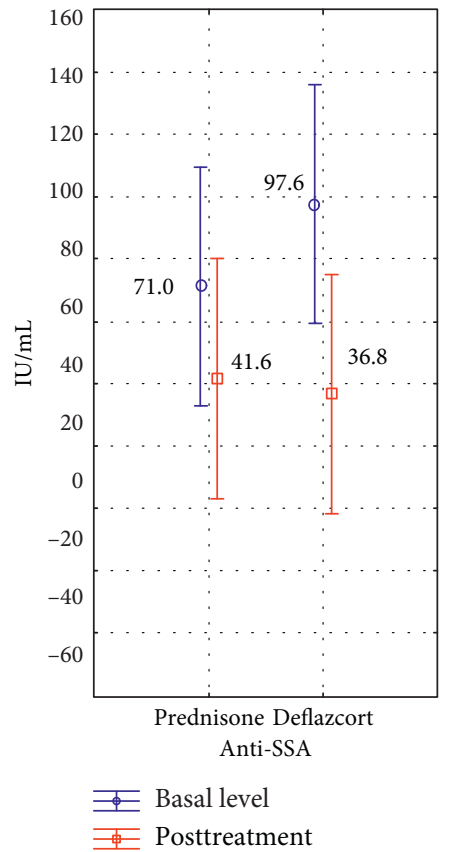

(a)

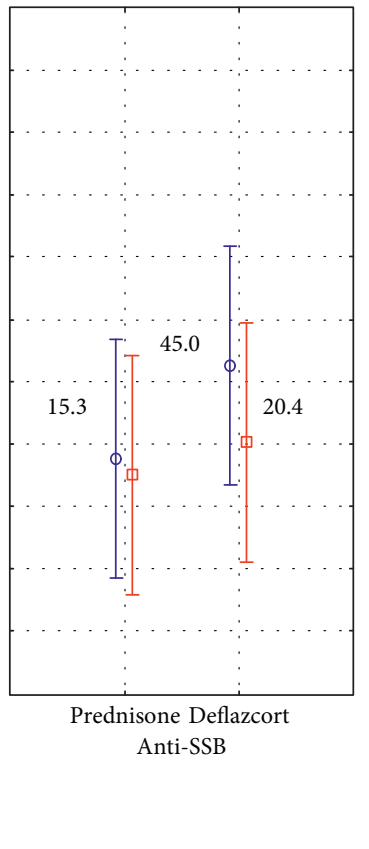

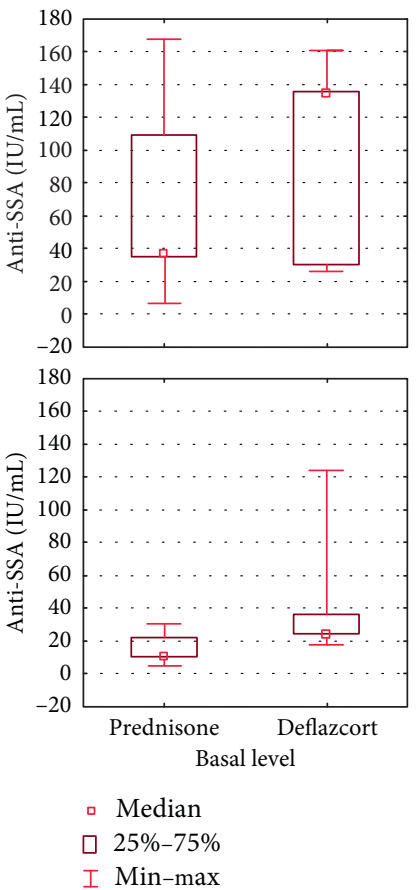
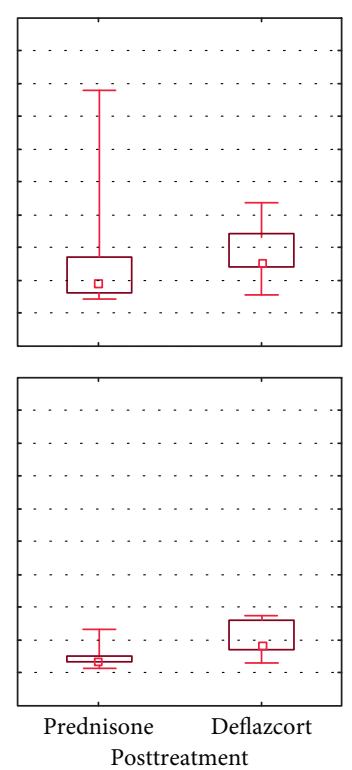

(b)

FIGURE 2: (a) Variance analysis of the mean in anti-SSA and anti-SSB antibodies before and after steroid treatment. (b) Comparative analysis of the median response of the serum level of autoantibodies, ranges, and interquartile ranges according to the indicated treatment and titration phase. Follow-up was done three months after the initial treatment.

greater frequency of attention of this pathology in our service. It was evidenced in the results that there is a decrease in serum levels of autoantibodies in the three diseases regardless of the type of steroids used since the comparison between prednisone and deflazacort showed no statistical significance. The decrease of autoantibodies is seen in the 


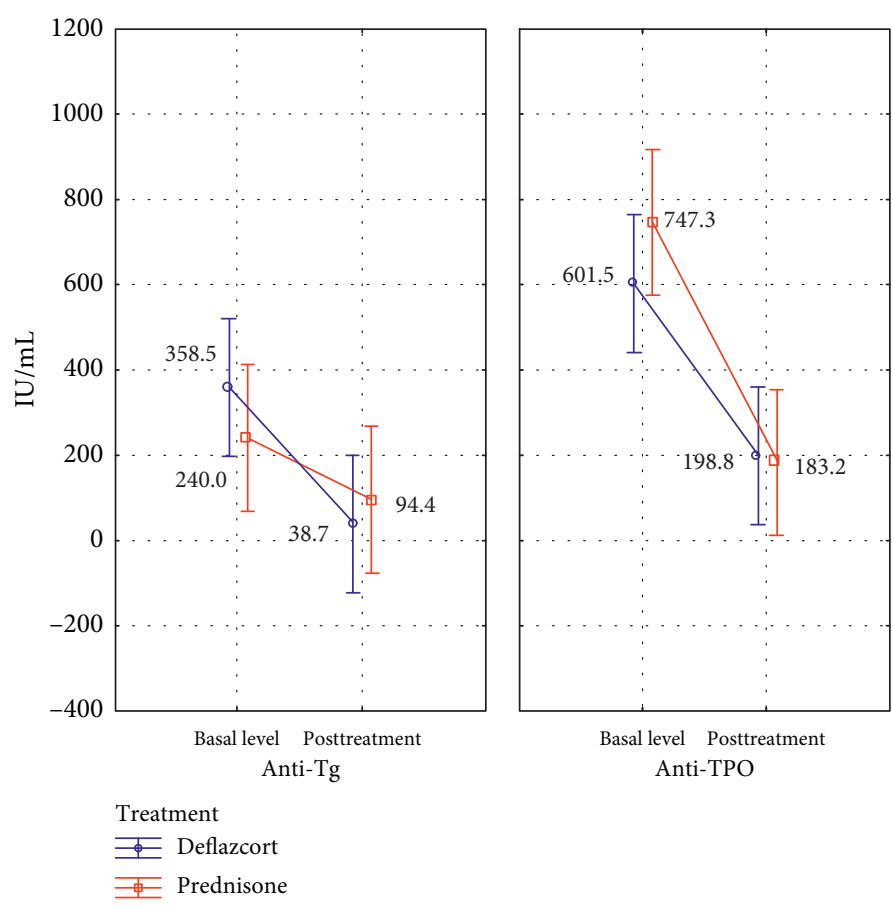

(a)

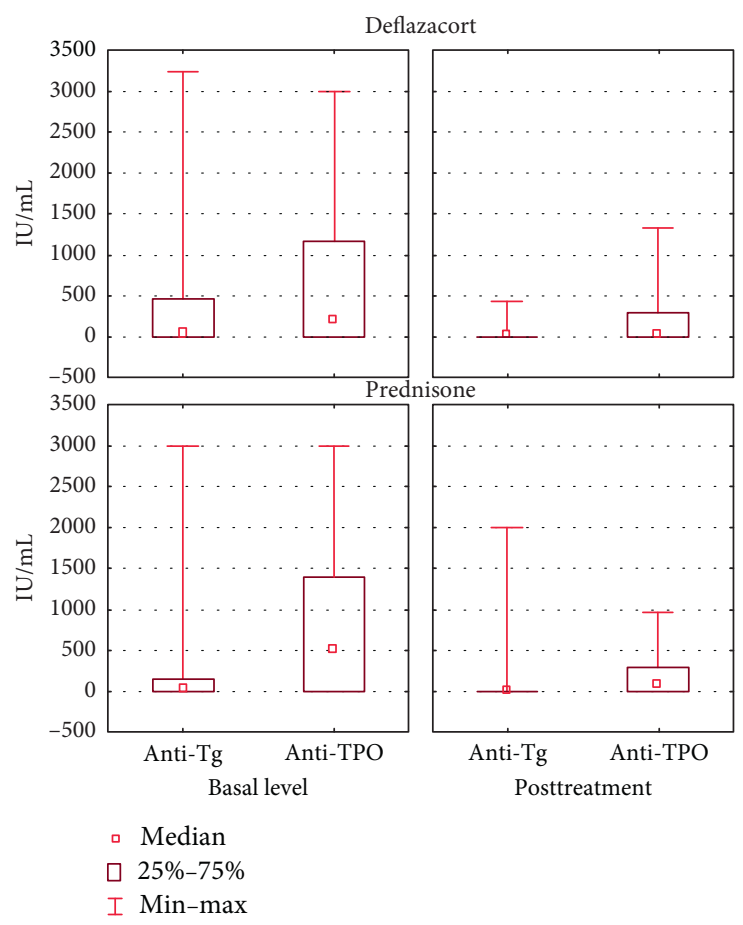

(b)

Figure 3: (a) Variance analysis of the mean in anti-Tg and anti-TPO antibodies before and after steroid treatment. (b) Comparative analysis of the median response of the serum level of autoantibodies, ranges, and interquartile ranges according to the indicated treatment and titration phase. Follow-up was done three months after the initial treatment.

study in the posttreatment of the diseases compared to the pretreatment.

There is sufficient evidence that demonstrates the safety in the use of alternate-day corticosteroid therapy in patients who may need long-term treatment with these medications. Suda et al. demonstrated that there is a $50 \%$ decrease in the infection rate in patients with rheumatoid arthritis with intake of steroids in alternate day versus the patients with daily intake for a period of one year [18]. There is no published evidence that objectively checks whether the steroid scheme on alternate days directly modifies the serum level of autoantibodies in patients with autoimmune diseases, so our results show that treatment on alternate days serves its purpose.

This clinical practice is based on basic immunological principles described since 1960, in articles published by several researchers and which unfortunately seem to be forgotten despite the results they shown.

In spite of the favourable results observed in this study, we consider it very important to complement with other studies that assess the clinical evolution of patients since it has been shown in other publications that with alternate-day corticosteroid therapy, the patients present less adverse effects.

The main reason for proposing the administration of steroids on alternate days is to reduce the prevalence of side effects including complications associated with suppression of the pituitary-hypothalamus-adrenal axis, improving the quality of life of patients with autoimmune diseases.
It is important to mention that the clinical activity of autoimmune disease does not correlate directly with serum levels of autoantibodies although these are the objective expression of autoimmunity [19].

\section{Conclusion}

Serum levels of autoantibodies in SLE, SS, and HT decrease significantly with alternate-day corticosteroid therapy. It is necessary to carry out comparative studies between daily and alternate day schemes jointly assessing the adverse effects, the decrease of autoantibodies, and the clinical activity of autoimmune disease. The use of steroids on alternate days instead of daily administration demonstrates a decrease in the adverse effects of the treatment and also a decrease in serum levels of autoantibodies.

\section{Abbreviations}

Anti-ds DNA: Double-stranded DNA autoantibodies

Anti-Tg: Anti-thyroglobulin autoantibodies

Anti-TPO: Anti-thyroid peroxidase autoantibodies

SLE: $\quad$ Systemic lupus erythematosus

SS: $\quad$ Sjögren syndrome

HT: $\quad$ Hashimoto's thyroiditis.

\section{Data Availability}

The research ethics committee restricts the patient's data used to support the findings of this study in order to protect 
patient privacy according to The Federal Law on Protection of Personal Data in Mexico. Data are available from Maria Eugenia Vargas Camaño (dra.maruvargascam@gmail.com) for researchers who met the criteria for access to confidential data.

\section{Additional Points}

(1) What is already known about this topic? The alternateday corticosteroid therapy reduces the adverse effects in the long-term treatment compared to the daily dose schedule. (2) What does this article add to our knowledge? The evidence of decreased serum autoantibody levels with treatment for three months. (3)How does this study impact current management guidelines? The relevance of this paper lies in being able to recommend this treatment as an alternative to short steroid cycles in autoimmune diseases that require it.

\section{Disclosure}

The study was carried out with the resources of the public health care institution.

\section{Conflicts of Interest}

All authors declare no conflicts of interest.

\section{Acknowledgments}

This work was not funded, but the resources used for research are those destined for the daily care of patients in the National Medical Center "20 de Noviembre", Instituto de Seguridad y Servicios Sociales de los Trabajadores del Estado. Special thanks are due to Luis Emmanuel Alonso-Bello for the work with the graphics.

\section{References}

[1] M. Cohn, "On the opposing views of the self-nonself discrimination by the immune system," Immunology of Cell Biology, vol. 87, no. 2, pp. 113-119, 2009.

[2] P. Sawla, A. Hossain, B. H. Hahn, and R. P. Singh, "Regulatory $t$ cells in systemic lupus erythematosus (SLE); Role of peptide tolerance," Autoimmunity Reviews, vol. 11, no. 9, pp. 611-614, 2012.

[3] P. Invernizzi and M. E. Gershwin, "The genetics of human autoimmune disease," Journal of Autoimmunity, vol. 33, no. 34, pp. 290-299, 2009.

[4] R. S. McHugh and E. M. Shevach, "The role of suppressor t cells in regulation of immune responses," Journal of Allergy and Clinical Immunology, vol. 110, no. 5, pp. 693-702, 2002.

[5] A. V. Miller and S. K. M. Ranatunga, "Immunotherapies in rheumatologic disorders," Medical Clinics of North America, vol. 96, no. 3, pp. 475-496, 2012.

[6] A. P. Grammatikos and G. C. Tsokos, "Immunodeficiency and autoimmunity: lessons from systemic lupus erythematosus," Trends in Molecular Medicine, vol. 18, no. 2, pp. 101-108, 2012.

[7] C. Yildirim-Toruner and B. Diamond, "Current and novel therapeutics in the treatment of systemic lupus erythematosus," Journal of Allergy and Clinical Immunology, vol. 127, no. 2, pp. 303-312, 2011.
[8] C. M. Spies, C. Strehl, M. C. Van Der Goes, J. W. J. Bijlsma, and F. Buttgereit, "Glucocorticoids," Best Practice \& Research Clinical Rheumatology, vol. 25, no. 6, pp. 891-900, 2011.

[9] E. Sarnes, L. Crofford, M. Watson, G. Dennis, H. Kan, and D. Bass, "Incidence and US costs of corticosteroid-associated adverse events: a systematic literature review," Clinical Therapeutics, vol. 33, no. 10, pp. 1413-1432, 2011.

[10] A. K. McDonough, J. R. Curtis, and K. G. Saag, "The epidemiology of glucocorticoid-associated adverse events," Current Opinion in Rheumatology, vol. 20, no. 2, pp. 131-137, 2008.

[11] D. M. Poetker and D. D. Reh, "A comprehensive review of the adverse effects of systemic corticosteroids," Otolaryngologic Clinics of North America, vol. 43, no. 4, pp. 753-768, 2010.

[12] D. Huscher, K. Thiele, E. Gromnica-Ihle et al., "Dose-related patterns of glucocorticoid-induced side effects," Annals of the Rheumatic Diseases, vol. 68, no. 7, pp. 1119-1124, 2009.

[13] J. N. Hoes, J. W. G. Jacobs, M. Boers et al., "Eular evidencebased recommendations on the management of systemic glucocorticoid therapy in rheumatic diseases," Annals of the Rheumatic Diseases, vol. 66, no. 12, pp. 1560-1567, 2007.

[14] R. R. MacGregor, J. N. Sheagren, M. B. Lipsett, and S. M. Wolff, “Alternate-day prednisone therapy," New England Journal of Medicine, vol. 280, no. 26, pp. 1427-1431, 1969.

[15] M. E. Carter and V. H. James, "Effect of alternate-day, singledose, corticosteroid therapy on pituitary-adrenal function," Annals of the Rheumatic Diseases, vol. 31, no. 5, pp. 379-383, 1972.

[16] M. Abbas, U. Sham-Una, T. Rambod, H. Niloofar, R. Ali, and Z. M. Hadi, "The effect of long-term steroid therapy on linear growth of nephrotic children," Iranian Journal of Pediatrics, vol. 21, no. 1, pp. 21-27, 2011.

[17] H. G. Morris, I. Neuman, and E. F. Ellis, "Plasma steroid concentrations during alternate-day treatment with prednisone," Journal of Allergy and Clinical Immunology, vol. 54, no. 6, pp. 350-358, 1974.

[18] M. Suda, S. Ohde, T. Tsuda, M. Kishimoto, and M. Okada, "Safety and efficacy of alternate-day corticosteroid treatment as adjunctive therapy for rheumatoid arthritis: a comparative study," Clinical Rheumatology, vol. 37, no. 8, pp. 2027-2034, 2018.

[19] K. Garber, "Immunology: a tolerant approach," Nature, vol. 507, no. 7493, pp. 418-420, 2014. 